\title{
Swift monitoring of the massive X-ray binary SAX J0635.2+0533
}

\author{
Nicola La Palombara and Sandro Mereghetti
}

\begin{abstract}
INAF, Istituto di Astrofisica Spaziale e Fisica Cosmica Milano, via E. Bassini 15, 20133 Milano, Italy e-mail: nicola@iasf-milano.inaf.it
\end{abstract}

Received 29 March 2017 / Accepted 6 May 2017

\begin{abstract}
SAX J0635.2+0533 is a binary pulsar with a very short pulsation period $(P=33.8 \mathrm{~ms})$ and a high long-term spin down $(\dot{P}>$ $3.8 \times 10^{-13} \mathrm{~s} \mathrm{~s}^{-1}$ ), which suggests a rotation-powered (instead of an accretion-powered) nature for this source. While it was discovered at a flux level around $10^{-11} \mathrm{erg} \mathrm{cm}^{-2} \mathrm{~s}^{-1}$, between 2003 and 2004, this source was detected with XMM-Newton at an average flux of about $10^{-13} \mathrm{erg} \mathrm{cm}^{-2} \mathrm{~s}^{-1}$; moreover, the flux varied by over one order of magnitude on timescales of a few days, sometimes decreasing to below $3 \times 10^{-14} \mathrm{erg} \mathrm{cm}^{-2} \mathrm{~s}^{-1}$. Since both the rotation-powered and the accretion-powered scenarios have difficulties to explain these properties, the nature of SAX J0635.2+0533 is still unclear.Here we report on our recent long-term monitoring campaign on SAX J0635.2+0533 carried out with Swift, and on a systematic reanalysis of all the RXTE observations performed between 1999 and 2001. We found that during this time interval, the source remained almost always active at a flux level above $10^{-12} \mathrm{erg} \mathrm{cm}^{-2} \mathrm{~s}^{-1}$.
\end{abstract}

Key words. stars: individual: SAX J0635+0533 - X-rays: binaries

\section{Introduction}

SAX J0635.2+0533 (also known as PSR J0635+0533) is a very peculiar X-ray source. Its first detection, obtained with BeppoSAX in 1997 (Kaaret et al. 1999), revealed a 2-10 keV flux of $1.2 \times 10^{-11} \mathrm{erg} \mathrm{cm} \mathrm{cm}^{-2} \mathrm{~s}^{-1}$ and a hard power-law spectrum (photon index $\sim 1.5$ ) extending to $40 \mathrm{keV}$; based on these spectral properties and on the positional coincidence with a Be star, it was classified as a high-mass X-ray binary (HMXRB). The BeppoSAX data also revealed X-ray pulsations at $33.8 \mathrm{~ms}$ (Cusumano et al. 2000). This is the shortest pulsation period observed in a high-mass binary pulsar: a comparably short period in an HMXRB has only been detected in A0538-66, a transient $\mathrm{Be} / \mathrm{X}$-ray binary in the Large Magellanic Cloud with a pulse period of $69 \mathrm{~ms}$ (Skinner et al. 1982). Based on the pulse frequencies measured with RXTE in 1999, it was possible to set a lower limit $\left(\dot{P}>3.8 \times 10^{-13} \mathrm{~s} \mathrm{~s}^{-1}\right)$ on its long-term spin down (Kaaret et al. 2000). This slow-down rate is high enough for the corresponding rotational energy loss $\left(\dot{E}_{\text {rot }}=10^{45} 4 \pi^{2} \dot{P} / P^{3}=4 \times\right.$ $10^{38} \mathrm{erg} \mathrm{s}^{-1}$ ) to sustain the observed $X$-ray luminosity: in this interpretation, alternative to the mass-accretion scenario, the X-ray emission could be due to the shocks between the relativistic wind of the pulsar and the wind of the companion star. This emission mechanism has been invoked for other binary systems composed of a compact object (a neutron star in most cases) orbiting a massive star, such as PSR B1259-63 (Tam et al. 2015), LS I +61 ${ }^{\circ}$ 303 (Paredes-Fortuny et al. 2015), and LS 5039 (Takata et al. 2014). These binaries have also been observed at very high energies, up to the $\mathrm{TeV}$ range, thus proving that they are one of the main sites of particle acceleration in the Galaxy (Dubus 2015). It is interesting to note that SAX J0635.2+0533 was considered a candidate counterpart of the EGRET source 2EG J0635+0521/3EG J0634+0521 (Hartman et al. 1999), since its position is within the error box of this unidentified galactic $\gamma$-ray source. This EGRET source corresponds to the Fermi source 1FGL J0636.0+0458c (Abdo et al. 2010), which was not confirmed in the second (Nolan et al. 2012) and third (Acero et al. 2015) Fermi catalogues, however; very probably the source was undetected in these catalogues since its significance dropped below the threshold as a result of time variability, change in the diffuse model, or of the shift from unbinned to binned likelihood in the catalogue analysis procedure.

Between September 2003 and April 2004, SAX J0635.2+ 0533 was observed by XMM-Newton in two different periods, with eight pointings in September-October 2003 (epoch D) and another two pointings in March-April 2004 (epoch E, Mereghetti \& La Palombara 2009). The source was detected only in six pointings, showing a large variability in the different observations: between September and October 2003, the source flux varied by at least a factor 10 , with a rise or decay time of only a few days. The source spectrum was well fitted with an absorbed power law, and the average flux in the energy range $0.2-12 \mathrm{keV}$ was $f_{\mathrm{X}} \sim 1.5 \times 10^{-13} \mathrm{erg} \mathrm{cm}^{-2} \mathrm{~s}^{-1}$. The maximum flux detected in these observations was $\sim 5 \times 10^{-13} \mathrm{erg} \mathrm{cm}^{-2} \mathrm{~s}^{-1}$, a factor $>20$ smaller than that measured at the time of the discovery in 1997. For the other four observations we could only set an upper limit on the source flux, with a minimum upper-limit value $f_{\mathrm{X}}<3 \times 10^{-14} \mathrm{erg} \mathrm{cm}^{-2} \mathrm{~s}^{-1}$.

There are no precise estimates for the distance of SAX J0635.2+0533, but from the properties of the proposed optical counterpart, Kaaret et al. (1999) derived a range of 2.5$5 \mathrm{kpc}$; a distance far in excess $5 \mathrm{kpc}$ is unlikely, since this source is in the Galactic anti-centre direction. Assuming a conservative source distance $\mathrm{d}_{5}$ of $5 \mathrm{kpc}$, the average flux during the $X M M-N e w t o n$ observations corresponds to a luminosity of a few $10^{32} \mathrm{~d}_{5}^{2} \mathrm{erg} \mathrm{s}^{-1}$, a value that is very low compared to the classical $\mathrm{Be} /$ neutron stars systems. 
Owing to the low luminosity and short spin period, direct accretion of matter onto the neutron star surface would require a magnetic field weaker than $B \sim 10^{8} \mathrm{G}$, at least three orders of magnitude lower than that expected in a young neutron star. If instead SAX J0635.2+0533 has a typical magnetic field, the low luminosity observed with XMM-Newton could be explained with mass accretion that stopped at the magnetospheric radius (Campana et al. 1995). However, this scenario is difficult to reconcile with the higher luminosity state observed with BeppoSAX and RXTE, since the pulsations with a relatively high pulsed fraction are unlikely when the magnetic centrifugal barrier operates.

The alternative scenario of a rotation-powered neutron star also presents some problems. The large flux variability seen in September 2003 can only be explained if the source was near to periastron on a very eccentric orbit, where great changes in the shock properties can be expected. Moreover, if the system indeed contains a neutron star with $\dot{E}_{\text {rot }}$ greater than a few $10^{38} \mathrm{erg} \mathrm{s}^{-1}$, a higher pulsar luminosity would be expected, since the typical efficiency of conversion of rotational energy into X-ray luminosity is of the order of $10^{-3}$, as observed in the cases of PSR B1259-63 (Chernyakova et al. 2006, 2009) and PSR B1957+20 (Huang \& Becker 2007).

In summary, SAX J0635.2+0533 is a unique source among the HMXRBs. Its luminosity is unusually low, both in quiescence and during outbursts. Because of these properties, both the accretion-powered and the rotation-powered scenarios imply several peculiarities with respect to other known HMXRBs. In order to investigate the long- and short-term behaviour of this source, we performed a monitoring campaign with Swift between 2015 and 2016 and analysed in a systematic way all the observations performed with RXTE between 1999 and 2001. In this way, we were able to obtain the overall long-term light curve of this source and study its variability pattern. Our aim was to investigate whether this source is characterized by prolonged periods of activity or if it spends most of the time in a quiescent state, interrupted only sporadically by bright (possibly periodic) outbursts.

\section{Observations and data analysis}

\subsection{Swift}

SAX J0635.2+0533 was observed repeatedly with Swift: 31 different pointings were performed between November 2015 and May 2016 (epoch F); then, after a time gap of three months, 2 additional pointings were performed in August 2016 (epoch G). We considered the data of the X-ray Telescope (XRT), which were obtained in photon-counting mode (Gehrels et al. 2004). In Table A.1 we report the date and effective exposure time of each observation.

We ignored pointing 28 , since it was too short $(\sim 100 \mathrm{~s})$ for a meaningful investigation. For each of the other pointings we retrieved the reduced files from the Swift archive $^{1}$ that were produced with the standard XRTPIPELINE processing routine. To study the source variability, we analysed the 32 data-sets separately; for each of them we performed a source detection to obtain an accurate evaluation of the source count rate (CR) and significance. We considered the source detected if its signal-tonoise ratio was above a threshold value of $2 \sigma$ c.l.; we adopted such a loose detection criterion considering that the presence

1 http://www.swift.ac.uk/swift_live/index.php

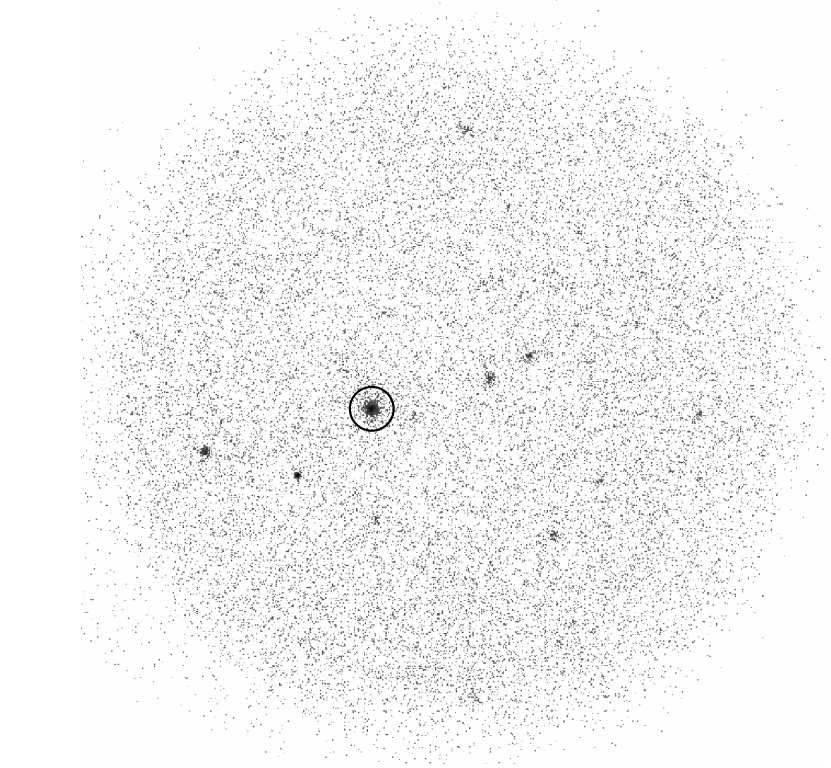

Fig. 1. The sky region around the position of SAX J0635.2+0533 (black circle), obtained by summing the $22 \mathrm{Swift} / \mathrm{XRT}$ observation where the source was detected; the sky region has a radius of $13 \mathrm{arcmin}$, while the black circle has a radius of 47 arcsec.

of a source at this position was already known. We found that SAX J0635.2+0533 was detected in 22 observations, while it was undetected in the first 6 observations (December 2015) and in another 4 observations during 2016; in case of a nondetection, we set a $3 \sigma$ c.l. upper limit on the CR (Table A.1).

In order to investigate the average spectral properties of the source, we used XSELECT to merge the event lists of the $22 \mathrm{ob}-$ servations where the source was detected and to accumulate an image of the field-of-view (reported in Fig. 1). In the total observing time of $\sim 74 \mathrm{ks}$, SAX J0635.2+0533 was detected with an average $\mathrm{CR}=(1.58 \pm 0.05) \times 10^{-2} \mathrm{cts} \mathrm{s}^{-1}$. To minimize the background contribution, we extracted the source spectrum by selecting events in a circular region of 20 pixel radius, corresponding to $\sim 47^{\prime \prime}$; the background spectrum was accumulated from a large circular area with no sources and radius of 100 pixels. We generated a cumulative exposure image of the merged observations and then used the task XRTMKARF to calculate the ancillary response file. The total spectrum was rebinned with a minimum of 20 counts per bin, and fitted in the energy range 0.3-10 keV using XSPEC 12.7.0. We obtained a good fit with an absorbed power law (Fig. 2), yielding a hydrogen column density $N_{\mathrm{H}}=\left(2.7_{-0.5}^{+0.6}\right) \times 10^{22} \mathrm{~cm}^{-2}$ and a photon index $\Gamma=2.0_{-0.3}^{+0.2}$. The average absorbed flux in the energy range $0.3-10 \mathrm{keV}$ is $f_{\mathrm{X}}=\left(1.08_{-0.08}^{+0.04}\right) \times 10^{-12} \mathrm{erg} \mathrm{cm}^{-2} \mathrm{~s}^{-1}$, while the corresponding unabsorbed flux is $2.5 \times 10^{-12} \mathrm{erg} \mathrm{cm}^{-2} \mathrm{~s}^{-1}$. Acceptable fits were also obtained with a thermal bremsstrahlung $(k T=6.5 \mathrm{keV})$ and with a blackbody $(k T \simeq 1 \mathrm{keV})$, although in the last case there are large residuals at high energies.

To also investigate the possible spectral dependence on the source flux, we accumulated two separate spectra for the observations with a source $\mathrm{CR}>0.02 \mathrm{cts} \mathrm{s}^{-1}$ and those with a lower $\mathrm{CR}$, respectively; in this way, we obtained two spectra with a similar count statistics. We performed a simultaneous fit of the two spectra with an absorbed power-law model, assuming a common hydrogen column density in order to avoid any degeneracy with the photon index. In Fig. 3 we report for both spectra the contour plot between the normalization and the photon 


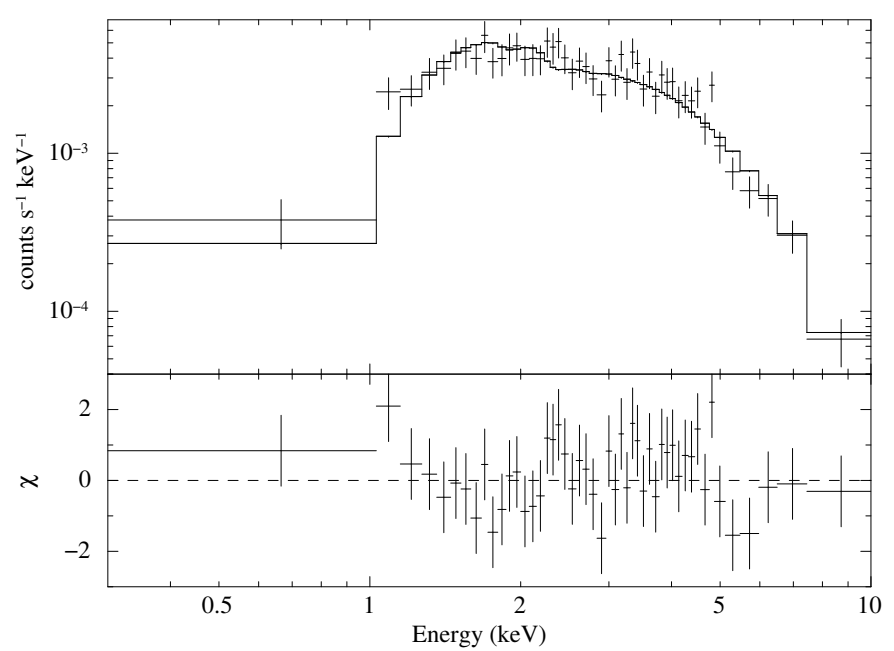

Fig. 2. Top panel: average spectrum of SAXJ0635.2+0533 with the best-fit power-law model. Bottom panel: data-model residuals in units of $\sigma$.

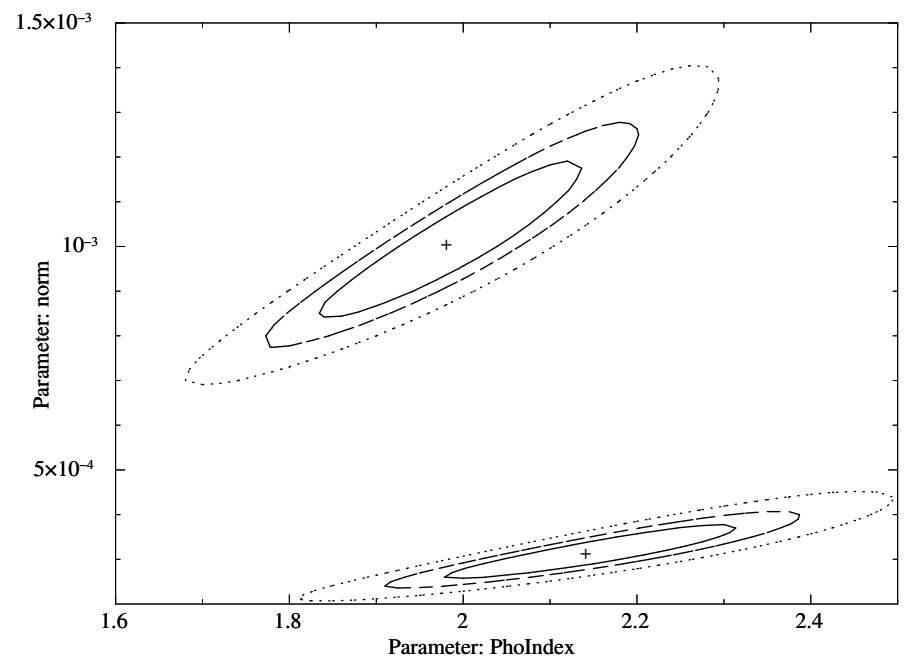

Fig. 3. Contour plots between the power-law photon index and normalization in the case of the highest and the lowest flux levels for SAX J0635.2+0533.

index, which shows no evidence for a variation in the photon index within the uncertainties. Using the flux/CR ratio obtained for the total spectrum, we converted the measured CR of each observation (or, in the case of missing detection, its upper limit at a $3 \sigma$ c.1.) into the corresponding energy flux; they are reported in Fig. 5.

We also performed a search for long-term periodicities in the Swift data by applying the Lomb-Scargle method (Scargle 1982). In Fig. 4 we report as an example the power distribution obtained by searching 1000 independent periods. In this case, a power $>11.5$ is necessary to claim a signal detection at $99 \%$ c.l.; on the other hand, the highest peak in our diagram, with power $\simeq 5$, has a probability higher than $99.9 \%$ to be obtained in the absence of a periodic signal. This documents that we found no evidence of a periodic signal; in particular, with the available data it is not possible to confirm the proposed orbital period of 11.2 days (Kaaret et al. 2000).

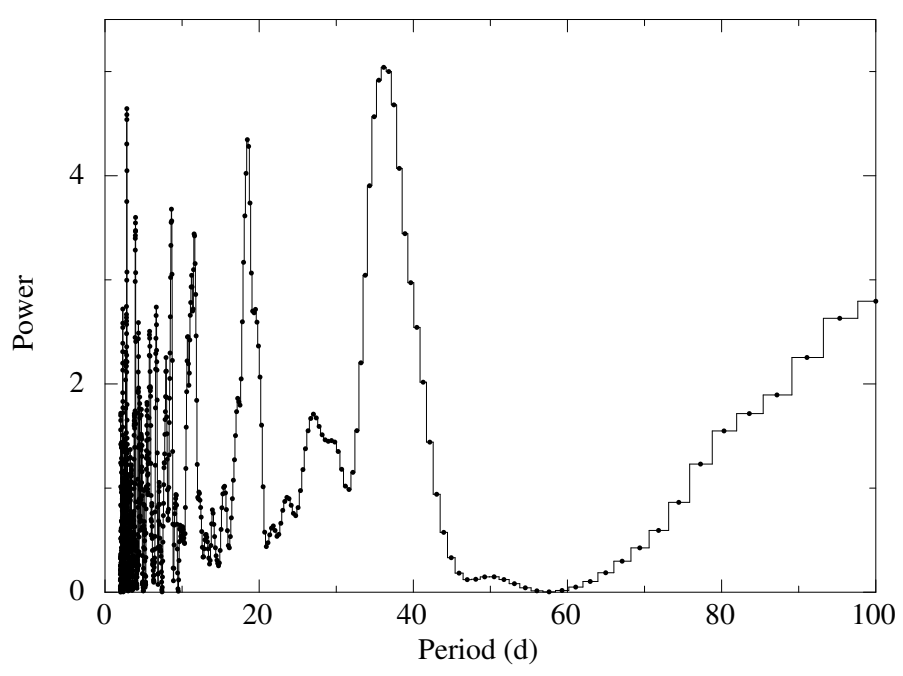

Fig. 4. Periodogram of SAX J0635.2+0533 obtained through a LombScargle analysis of the Swift data obtained between 2015 and 2016.

\subsection{RXTE}

RXTE (Bradt et al. 1993) observed SAX J0635.2+0533 in three different epochs between 1999 and 2001, with 13 pointings in August-September 1999 (epoch A), 5 pointings in May 2000 (epoch B), and 49 pointings between November 2000 and January 2001 (epoch C); in Table A.2 we report the dates and effective exposure times of each observation. Only the results of epoch A were published (Kaaret et al. 2000). We therefore analysed all the observations of the three epochs in the same way. We considered only the data of the Proportional Counter Array (PCA), which covers the energy range $2-100 \mathrm{keV}$ and comprises five identical coalligned gas-filled Proportional Counter Units (PCUs). The exposures of the individual observations were between 1.6 and $6.9 \mathrm{ks}$, and during each of them, between 2 and 5 PCUs were active.

For the spectral analysis we considered the Standard 2 data and applied the procedure suggested by the instrument team ${ }^{2}$. We separately analysed each observation; moreover, within each observation, we separately analysed the time intervals characterized by a different number of active PCUs (if any). In each case we created the applicable Good Time Interval and extracted the source and background spectra using only the PCU top layer, which gives a higher signal-to-noise ratio. Then we corrected for the dead time and created the applicable response matrix.

Although the source was always detected, for most observations the spectrum had a low count statistics, which hampered an accurate spectral analysis. Therefore, we assumed an absorbed power-law model fixing both $N_{\mathrm{H}}$ and $\Gamma$ to the best-fit values obtained with Swift $\left(2.7 \times 10^{22} \mathrm{~cm}^{-2}\right.$ and 2 , respectively) and left only the normalization free to vary. In this way, we estimated the fluxes reported in Fig. 5.

We searched for the presence of the pulsations at $\sim 34 \mathrm{~ms}$ in the data of 2000 and 2001 (epochs B and C), which were not analysed by Kaaret et al. (2000). Following these authors, we used the top-layer data in the energy range 4.4-23.6 keV collected in Good Xenon format. The arrival times were barycentred using the position of the optical counterpart and analysed using the Rayleigh test statistics. To take into account a possible spin-up or spin-down of the pulsar since the time of the previous period measurement ( $P=33.9 \mathrm{~ms}$ in August-September 1999),

2 http://heasarc.gsfc.nasa.gov/docs/xte/recipes/pca_ spectra.html 

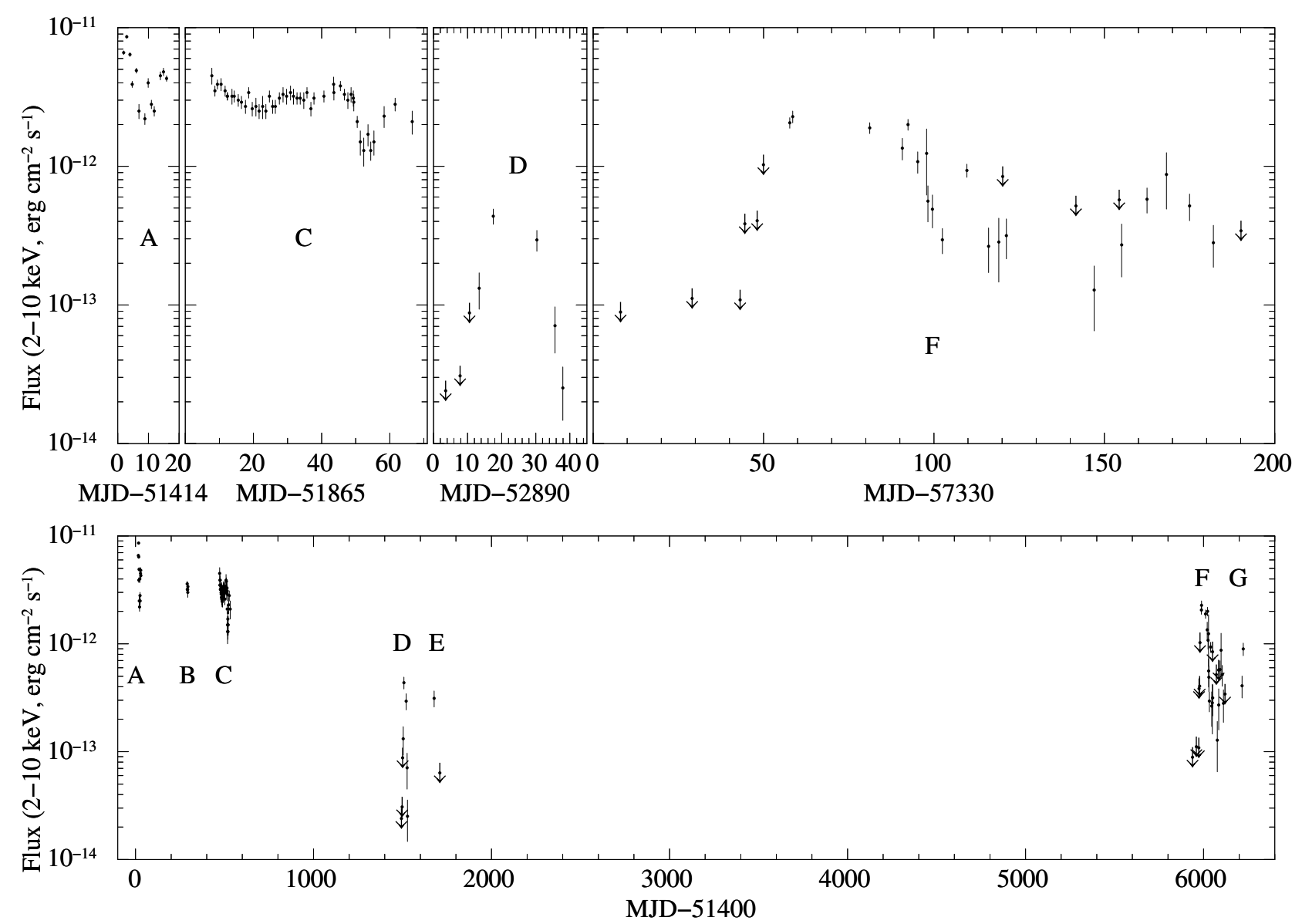

Fig. 5. Unabsorbed 2-10 keV flux of SAX J0635.2+0533 measured during the observations performed with RXTE (epochs A, B, and C), XMMNewton (epochs D and E), and Swift (epochs F and G) between 1999 and 2016. Upper panels: source light curve for the observations of epochs A, C, D, and F. Lower panel: overall light curve since MJD = 51400 (August 10, 1999).

we searched for periods in a relatively wide range, from 33.4 to $34.4 \mathrm{~ms}$. This corresponds to a conservative assumption of $|\dot{P}| \sim$ $10^{-11} \mathrm{~s} \mathrm{~s}^{-1}$ for the maximum spin-up/spin-down of the source. We performed the period search in each observation separately, without finding any significant excess in the power distributions; in all cases we found distribution peaks with power $Z^{2}<23$, corresponding to a chance detection probability $P>3.8 \%$. We also searched for excess power in the periodograms summed over groups of observations, again with negative results. We note, however, that the frequency modulation induced by orbital motion reduces the sensitivity of this analysis. Unfortunately, the orbital parameters derived by Kaaret et al. (2000) are not sufficiently precise to correct the arrival times for the orbital motion. After accounting for the large contribution of the background counts, we estimate that the single observations give a sensitivity to pulsed fractions of the order of $40-60 \%$, with the exact value depending on their duration and source flux.

\section{Discussion}

In Fig. 5 (lower panel) we report the long-term light curve of SAX J0635.2+0533 since MJD = 51400 (August 10, 1999); it includes all the flux measurements obtained after the source discovery in 1997 with different telescopes. In the four upper panels we show a zoom into the observations of epochs A, C, D, and $\mathrm{F}$, respectively; for each of them we use the same scale for the time and flux axes in order to better compare the flux variability among the different epochs. The light curve shows that
SAX J0635.2+0533 was clearly detected in most of the observations, although at very different flux levels. The source is very variable, with a dynamic range of more than two orders of magnitude; moreover, this variability is also rather fast, since the source flux can increase or decrease by one order of magnitude over a timescale of a few days. However, in spite of this high variability, we never again observed an outburst as luminous as that seen with BeppoSAX in 1997. Therefore, this type of event must be very rare.

In most observations the source was detected at a flux level

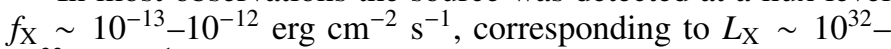
$10^{33} \mathrm{erg} \mathrm{s}^{-1}$. In the latest years, similar quiescent luminosities have been observed in several Be X-ray binaries, and various emission mechanisms have been proposed to explain them (see e.g. Tsygankov et al. 2017b). In the case of SAX J0635.2+0533, we can exclude that the observed low luminosity originates from the companion: in fact, although the intrinsic X-ray luminosity of Be stars can be as high as $\sim 10^{32} \mathrm{erg} \mathrm{s}^{-1}$ (Nazé 2009; Nazé et al. 2011), these stars do not show the high and fast variability observed in SAX J0635.2+0533. For the same reason, thermal emission from the neutron star due to crustal heating in previous outbursts cannot be considered either. Moreover, based on the relation between quiescent luminosity and average accretion rate, $L_{\mathrm{q}}=\left(\dot{M} / 10^{-11} M_{\odot} \mathrm{yr}^{-1}\right) \times 6 \times 10^{32} \mathrm{erg} \mathrm{s}^{-1}$ (Tsygankov et al. 2017b), we would expect $L_{\mathrm{q}} \lesssim 10^{31} \mathrm{erg} \mathrm{s}^{-1}$, a value well below those observed in SAX J0635.2+0533. Therefore we conclude that the source emission is most likely due to matter accretion. However, the very short pulse period of $33.8 \mathrm{~ms}$ 


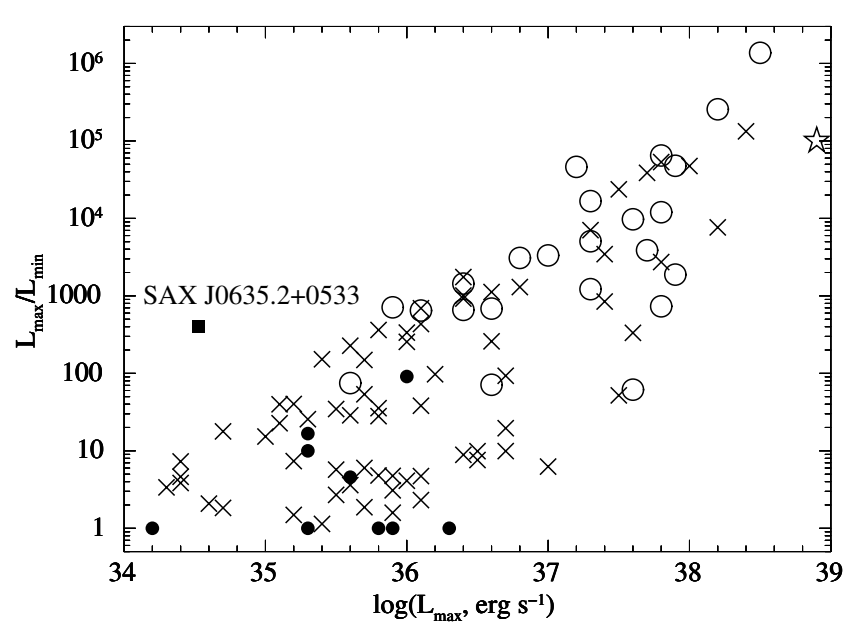

Fig. 6. X-ray luminosity ratio $L_{\max } / L_{\min }$ as a function of $L_{\max }$, for SAX J0635.2+0533 (filled square), A0538-66 (star), the persistent BeXRBs (filled circles), and the transient BeXRBs in the MW (open circles) and in the SMC (crosses).

places several constraints on the accretion regime: on one hand, it hampers any possibility of accretion from a cold recombined disc, regardless of the magnetic field (Tsygankov et al. 2017a); on the other hand, it makes subsonic accretion highly unlikely, even in the case of plasma radiative cooling (Shakura et al. 2013). For a typical neutron star magnetic field $\left(B \sim 10^{12}\right)$, the low source luminosity can only be explained with a propeller regime, where the accreting matter is stopped by the centrifugal barrier at the magnetosphere: this is true not only for the lowluminosity states, but even for the outburst observed in 1997.

A high flux variability is rather unusual for a low-luminosity BeXRB such as SAX J0635.2+0533, where the maximum flux observed in 1997 implies $L_{\max } \simeq 3.4 \times 10^{34} \mathrm{erg} \mathrm{s}^{-1}$. From this point of view, it is interesting to compare this source with other known BeXRBs, both in the Milky Way (MW) and in the Small Magellanic Cloud (SMC). In Fig. 6 we report the $L_{\max } / L_{\min }$ ratio as a function of $L_{\max }$ for several known BeXRBs: open circles represent the transient MW sources (Tsygankov et al. 2017b), while the crosses refer to the SMC sources reported by Haberl \& Sturm (2016); in addition, filled circles represent the class of persistent and low-luminosity BeXRBs with long spin periods (see e.g. La Palombara et al. 2012); finally, we also report (as star) the short-period $(P=69 \mathrm{~ms})$ binary pulsar in the LMC A0538-66 (Skinner et al. 1982; Kretschmar et al. 2004). The figure shows that the variability properties as a function of maximum luminosity for the MW and MC sources are similar: in both cases the source dynamic range increases with the maximum luminosity, while the low-luminosity sources are also less variable (as in the case of the persistent sources). The lack of persistent high-luminosity sources (lower right corner of the figure) is due to the transient nature of the BeXRBs, which only show a high luminosity during the periastron passage of the NS (type I outbursts) or during a massive matter ejection from the Be star (type II outbursts).

In Fig. 6, SAX J0635.2+0533 (indicated as a filled square) is clearly an outlier: it shows the greatest dynamic range $\left(L_{\max } / L_{\min } \simeq 400\right)$ of the less luminous sources. Although the lack of SMC sources in the same region of the plot might be due to the difficulty of detecting low-luminosity sources at such a large distance ${ }^{3}$, this caveat does not apply to the sources in the Milky Way. This confirms that SAX J0635.2+0533 has rather peculiar properties at variance with those of the normal accretionpowered BeXRBs.

Acknowledgements. We acknowledge the constant support of the Swift team for the execution of the source monitoring between 2015 and 2016.

\section{References}

Abdo, A. A., Ackermann, M., Ajello, M., et al. 2010, ApJS, 188, 405 Acero, F., Ackermann, M., Ajello, M., et al. 2015, ApJS, 218, 23 Bradt, H. V., Rothschild, R. E., \& Swank, J. H. 1993, A\&AS, 97, 355

Campana, S., Stella, L., Mereghetti, S., \& Colpi, M. 1995, A\&A, 297, 385

Chernyakova, M., Neronov, A., Lutovinov, A., Rodriguez, J., \& Johnston, S. 2006, MNRAS, 367, 1201

Chernyakova, M., Neronov, A., Aharonian, F., Uchiyama, Y., \& Takahashi, T. 2009, MNRAS, 397, 2123

Cusumano, G., Maccarone, M. C., Nicastro, L., Sacco, B., \& Kaaret, P. 2000, ApJ, 528, L25

Dubus, G. 2015, Comptes Rendus Physique, 16, 661

Gehrels, N., Chincarini, G., Giommi, P., et al. 2004, ApJ, 611, 1005

Haberl, F., \& Sturm, R. 2016, A\&A, 586, A81

Hartman, R. C., Bertsch, D. L., Bloom, S. D., et al. 1999, ApJS, 123, 79

Huang, H. H., \& Becker, W. 2007, A\&A, 463, L5

Kaaret, P., Cusumano, G., \& Sacco, B. 2000, ApJ, 542, L41

Kaaret, P., Piraino, S., Halpern, J., \& Eracleous, M. 1999, ApJ, 523, 197

Kretschmar, P., Wilms, J., Staubert, R., Kreykenbohm, I., \& Heindl, W. A. 2004 , in 5th INTEGRAL Workshop on the INTEGRAL Universe, ed. V. Schoenfelder, G. Lichti, \& C. Winkler, ESA SP, 552, 329

La Palombara, N., Sidoli, L., Esposito, P., Tiengo, A., \& Mereghetti, S. 2012, A\&A, 539, A82

Mereghetti, S., \& La Palombara, N. 2009, A\&A, 504, 181

Nazé, Y. 2009, A\&A, 506, 1055

Nazé, Y., Broos, P. S., Oskinova, L., et al. 2011, ApJS, 194, 7

Nolan, P. L., Abdo, A. A., Ackermann, M., et al. 2012, ApJS, 199, 31

Paredes-Fortuny, X., Ribó, M., Bosch-Ramon, V., et al. 2015, A\&A, 575, L6

Scargle, J. D. 1982, ApJ, 263, 835

Shakura, N., Postnov, K., \& Hjalmarsdotter, L. 2013, MNRAS, 428, 670

Skinner, G. K., Bedford, D. K., Elsner, R. F., et al. 1982, Nature, 297, 568

Takata, J., Leung, G. C. K., Tam, P. H. T., et al. 2014, ApJ, 790, 18

Tam, P. H. T., Li, K. L., Takata, J., et al. 2015, ApJ, 798, L26

Tsygankov, S. S., Mushtukov, A. A., Suleimanov, V. F., et al. 2017a, A\&A, submitted [arXiv: 1703.04528]

Tsygankov, S. S., Wijnands, R., Lutovinov, A. A., Degenaar, N., \& Poutanen, J. 2017b, MNRAS, submitted [arXiv: 1703.04634] 3 For SMC sources, the minimum detected flux is $f_{\text {min }} \lesssim$
$10^{-14} \mathrm{erg} \mathrm{cm}^{-2} \mathrm{~s}^{-1}$, which implies $L_{\min } \lesssim 4 \times 10^{33} \mathrm{erg} \mathrm{s}^{-1}$. 


\section{Appendix A: Summary of the Swift and RXTE observations}

Table A.1. Main parameters of the source observations performed with Swift.

\begin{tabular}{|c|c|c|c|c|c|}
\hline $\begin{array}{l}\text { Observation } \\
\text { Number }\end{array}$ & $\begin{array}{c}\text { Start observation } \\
\text { date - UT }\end{array}$ & $\begin{array}{l}\text { Net exposure } \\
(\mathrm{ks})\end{array}$ & $\begin{array}{c}\mathrm{CR} \\
\left(\times 10^{-3} \mathrm{cts} \mathrm{s}^{-1}\right)\end{array}$ & $\begin{array}{c}\text { Flux }(0.3-10 \mathrm{keV}) \\
\left(\times 10^{-13} \mathrm{erg} \mathrm{cm}^{-2} \mathrm{~s}^{-1}\right)\end{array}$ & $\begin{array}{c}\text { Luminosity }^{(a)}(0.3-10 \mathrm{keV}) \\
\left(\times 10^{33} \mathrm{erg} \mathrm{s}^{-1}\right)\end{array}$ \\
\hline 1 & $2015-11-12-00: 44: 56$ & 9.3 & $<1.4$ & $<1.0$ & $<0.6$ \\
\hline 2 & 2015-12-03-00:59:16 & 9.7 & $<1.8$ & $<1.2$ & $<0.8$ \\
\hline 3 & 2015-12-17-03:05:54 & 6.0 & $<1.7$ & $<1.2$ & $<0.8$ \\
\hline 4 & 2015-12-18-12:44:49 & 1.9 & $<6.1$ & $<4.2$ & $<2.8$ \\
\hline 5 & $2015-12-22-02: 44: 50$ & 3.8 & $<6.5$ & $<4.4$ & $<2.9$ \\
\hline 6 & $2015-12-23-23: 29: 48$ & 0.9 & $<16.4$ & $<11.1$ & $<7.3$ \\
\hline 7 & 2015-12-31-16:23:48 & 5.2 & $33 \pm 3$ & $22 \pm 2$ & $15 \pm 1$ \\
\hline 8 & 2016-01-01-13:09:41 & 3.8 & $36 \pm 3$ & $25 \pm 2$ & $16 \pm 2$ \\
\hline 9 & 2016-01-24-03:39:37 & 5.2 & $30 \pm 3$ & $21 \pm 2$ & $13 \pm 1$ \\
\hline 10 & $2016-02-02-17: 37: 57$ & 1.9 & $22 \pm 4$ & $15 \pm 3$ & $10 \pm 2$ \\
\hline 11 & 2016-02-04-09:27:21 & 5.0 & $32 \pm 3$ & $22 \pm 2$ & $14 \pm 1$ \\
\hline 12 & 2016-02-07-05:52:29 & 2.2 & $17 \pm 3$ & $12 \pm 2$ & $8 \pm 1$ \\
\hline 13 & 2016-02-09-20:37:15 & 0.4 & $20 \pm 10$ & $14 \pm 7$ & $9 \pm 4$ \\
\hline 14 & 2016-02-10-05:43:32 & 1.8 & $9 \pm 3$ & $6 \pm 2$ & $4 \pm 1$ \\
\hline 15 & 2016-02-11-13:38:18 & 3.1 & $8 \pm 2$ & $5 \pm 1$ & $3.5 \pm 0.9$ \\
\hline 16 & 2016-02-14-11:50:20 & 7.1 & $5 \pm 1$ & $3.2 \pm 0.7$ & $2.1 \pm 0.4$ \\
\hline 17 & 2016-02-21-16:07:04 & 8.1 & $15 \pm 2$ & $10 \pm 1$ & $6.7 \pm 0.7$ \\
\hline 18 & 2016-02-28-01:12:03 & 2.9 & $4 \pm 1$ & $3 \pm 1$ & $1.9 \pm 0.7$ \\
\hline 19 & 2016-03-02-01:00:09 & 1.5 & $5 \pm 2$ & $3 \pm 1$ & $2 \pm 1$ \\
\hline 20 & 2016-03-03-04:09:49 & 0.8 & $<13$ & $<9$ & $<6$ \\
\hline 21 & 2016-03-04-05:38:50 & 4.6 & $5 \pm 2$ & $3 \pm 1$ & $2.3 \pm 0.7$ \\
\hline 22 & 2016-03-24-15:32:05 & 3.4 & $<8$ & $<6$ & $<4$ \\
\hline 23 & 2016-03-30-00:45:50 & 4.2 & $2 \pm 1$ & $1.4 \pm 0.7$ & $0.9 \pm 0.4$ \\
\hline 24 & 2016-04-06-08:03:15 & 1.7 & $<9$ & $<6$ & $<4$ \\
\hline 25 & 2016-04-07-01:38:57 & 2.1 & $4 \pm 2$ & $3 \pm 1$ & $1.9 \pm 0.8$ \\
\hline 26 & 2016-04-14-12:15:17 & 3.7 & $9 \pm 2$ & $6 \pm 1$ & $4.1 \pm 0.8$ \\
\hline 27 & 2016-04-20-05:26:58 & 0.5 & $14 \pm 6$ & $9 \pm 4$ & $6 \pm 3$ \\
\hline 28 & $2016-04-21-15: 15: 29$ & 0.1 & - & - & - \\
\hline 29 & 2016-04-27-00:13:19 & 3.6 & $8 \pm 2$ & $6 \pm 1$ & $3.7 \pm 0.8$ \\
\hline 30 & 2016-05-04-00:03:16 & 3.2 & $4 \pm 1$ & $3 \pm 1$ & $2.0 \pm 0.7$ \\
\hline 31 & $2016-05-12-02: 28: 06$ & 4.4 & $<5$ & $<4$ & $<2$ \\
\hline 32 & 2016-08-16-06:16:08 & 3.9 & $7 \pm 2$ & $4 \pm 1$ & $2.9 \pm 0.7$ \\
\hline 33 & 2016-08-23-04:06:43 & 5.0 & $14 \pm 2$ & $10 \pm 1$ & $6.4 \pm 0.9$ \\
\hline
\end{tabular}

Notes. ${ }^{(a)}$ Corrected for the absorption, and assuming a distance of $5 \mathrm{kpc}$. Observations of epochs $\mathrm{F}$ and $\mathrm{G}$ are separated by a horizontal line.

Table A.2. Main parameters of the source observations performed with RXTE.

\begin{tabular}{|c|c|c|c|c|c|}
\hline $\begin{array}{c}\text { Observation } \\
\text { number }\end{array}$ & $\begin{array}{c}\text { Observation } \\
\text { ID }\end{array}$ & $\begin{array}{c}\text { Start observation } \\
\text { date - UT }\end{array}$ & $\begin{array}{c}\text { Net exposure } \\
\text { (s) }\end{array}$ & $\begin{array}{c}\text { Flux }(2-10 \mathrm{keV}) \\
\left(\times 10^{-12} \mathrm{erg} \mathrm{cm}^{-2} \mathrm{~s}^{-1}\right)\end{array}$ & $\begin{array}{c}\text { Luminosity }^{(a)}(2-10 \mathrm{keV}) \\
\left(\times 10^{33} \mathrm{erg} \mathrm{s}^{-1}\right)\end{array}$ \\
\hline 01 & 40131-01-01-00 & 1999-08-25 22:10:06.8 & 2896 & $6.6 \pm 0.2$ & $22.0 \pm 0.7$ \\
\hline 02 & $40131-01-02-00$ & 1999-08-26 22:06:28.2 & 2880 & $8.6_{-0.3}^{+0.2}$ & $28.7_{-1.0}^{+0.7}$ \\
\hline 03 & $40131-01-03-00$ & 1999-08-27 23:43:50.6 & 2640 & $6.4_{-0.3}^{+0.2}$ & $21.3_{-1.0}^{+0.7}$ \\
\hline 04 & 40131-01-04-00 & 1999-08-28 17:15:50.1 & 2800 & $3.9_{-0.3}^{+0.2}$ & $13.0_{-10}^{+0.7}$ \\
\hline 05 & $40131-01-05-00$ & 1999-08-30 01:12:46.7 & 2368 & $4.9_{-0.3}^{+0.2}$ & $16.3_{-1.0}^{+0.7}$ \\
\hline 06 & 40131-01-06-00 & 1999-08-30 21:57:38.6 & 2944 & $2.5_{-0.2}^{+0.3}$ & $8.3_{-0.7}^{+1.0}$ \\
\hline 07 & 40131-01-07-00 & 1999-09-01 20:35:06.1 & 2592 & $2.2_{-0.3}^{+0.2}$ & $7.3_{-10}^{+0.7}$ \\
\hline 08 & 40131-01-08-00 & 1999-09-02 21:48:36.9 & 2928 & $4.0_{-0.2}^{+0.3}$ & $13.3_{-0.7}^{+1.0}$ \\
\hline 09 & 40131-01-09-00 & 1999-09-03 23:32:07.3 & 2816 & $2.8 \pm 0.2$ & $9.3 \pm 0.7$ \\
\hline 10 & $40131-01-10-00$ & 1999-09-04 21:49:58.9 & 3120 & $2.5 \pm 0.2$ & $8.3 \pm 0.7$ \\
\hline 11 & $40131-01-11-00$ & 1999-09-06 21:51:07.2 & 3120 & $4.5_{-0.2}^{+0.3}$ & $15.0_{-0.7}^{+1.0}$ \\
\hline 12 & $40131-01-12-00$ & 1999-09-07 21:42:20.2 & 3184 & $4.8 \pm 0.3$ & $16.0 \pm 1.0$ \\
\hline 13 & 40131-01-13-00 & 1999-09-08 21:47:07.0 & 3168 & $4.3 \pm 0.2$ & $14.3 \pm 0.7$ \\
\hline
\end{tabular}

Notes. ${ }^{(a)}$ Corrected for the absorption, and assuming a distance of $5 \mathrm{kpc}$. Observations of epochs A, B, and C are separated by a horizontal line. 
Table A.2. continued.

\begin{tabular}{|c|c|c|c|c|c|}
\hline $\begin{array}{l}\text { Observation } \\
\text { number }\end{array}$ & $\begin{array}{l}\text { Observation } \\
\text { ID }\end{array}$ & $\begin{array}{c}\text { Start observation } \\
\text { date - UT }\end{array}$ & $\begin{array}{c}\text { Net exposure } \\
(\mathrm{ks})\end{array}$ & $\begin{array}{c}\text { Flux }(2-10 \mathrm{keV}) \\
\left(\times 10^{-12} \mathrm{erg} \mathrm{cm}^{-2} \mathrm{~s}^{-1}\right)\end{array}$ & $\begin{array}{c}\text { Luminosity }^{(a)}(2-10 \mathrm{keV}) \\
\left(\times 10^{33} \mathrm{erg} \mathrm{s}^{-1}\right)\end{array}$ \\
\hline 14 & $50085-01-01-00$ & $2000-05-2604: 18: 08.9$ & 6064 & $3.6 \pm 0.2$ & $12.0 \pm 0.7$ \\
\hline 15 & $50085-01-02-00$ & 2000-05-27 02:42:24.2 & 6480 & $3.2 \pm 0.2$ & $10.7 \pm 0.7$ \\
\hline 16 & 50085-01-03-00 & 2000-05-28 02:58:28.4 & 5704 & $3.2 \pm 0.2$ & $10.7 \pm 0.7$ \\
\hline 17 & 50085-01-04-00 & 2000-05-29 04:09:25.3 & 3248 & $3.0_{-0.2}^{+0.3}$ & $10.0_{-0.7}^{+1.0}$ \\
\hline 18 & 50085-01-05-00 & 2000-05-30 05:36:15.3 & 2832 & $3.4_{-0.2}^{+0.3}$ & $11.3_{-0.7}^{+1.0}$ \\
\hline 19 & $50085-01-06-00$ & $2000-11-24$ 18:36:26.8 & 1960 & $4.5_{-0.3}^{+0.6}$ & $15.0_{-10}^{+2.0}$ \\
\hline 20 & 50085-01-07-00 & 2000-11-25 16:47:27.2 & 2488 & $3.5_{-0.2}^{+0.3}$ & $11.7_{-0.7}^{+1.0}$ \\
\hline 21 & $50085-01-08-00$ & 2000-11-26 10:18:51.3 & 3104 & $3.9_{-0.4}^{+0.2}$ & $13.0_{-1.3}^{+0.0}$ \\
\hline 22 & 50085-01-09-00 & 2000-11-27 11:48:54.7 & 2352 & $3.9_{-0.3}^{+0.4}$ & $13.0_{-1.0}^{+1.3}$ \\
\hline 23 & 50085-01-10-00 & 2000-11-28 16:28:57.8 & 2016 & $3.5_{-0.4}^{+0.3}$ & $11.7_{-1.3}^{+1.0}$ \\
\hline 24 & 50085-01-11-00 & 2000-11-29 08:24:00.9 & 2432 & $3.2_{-0.3}^{+0.4}$ & $10.7_{-1.0}^{+0.7}$ \\
\hline 25 & $50085-01-12-00$ & 2000-11-30 16:18:04.6 & 2000 & $3.2_{-0.3}^{+0.4}$ & $10.7_{-1.0}^{+1.3}$ \\
\hline 26 & 50085-01-13-00 & 2000-12-01 08:10:08.8 & 3264 & $3.2 \pm 0.3$ & $10.7 \pm 1.0$ \\
\hline 27 & 50085-01-14-00 & 2000-12-02 12:51:11.5 & 3024 & $3.0 \pm 0.3$ & $10.0 \pm 1.0$ \\
\hline 28 & $50085-01-15-00$ & 2000-12-03 11:08:20.8 & 2664 & $2.9 \pm 0.3$ & $9.7 \pm 1.0$ \\
\hline 29 & 50085-01-16-00 & 2000-12-04 15:50:21.4 & 2032 & $2.7_{-0.4}^{+0.3}$ & $9.0_{-1.3}^{+1.0}$ \\
\hline 30 & $50085-01-17-00$ & 2000-12-05 14:04:06.8 & 2448 & $3.4_{-0.4}^{+0.4}$ & $11.3_{-1.3}^{+1.0}$ \\
\hline 31 & 50085-01-18-00 & 2000-12-06 15:36:32.0 & 2544 & $2.6 \pm 0.3$ & $8.7 \pm 1.0$ \\
\hline 32 & 50085-01-19-00 & 2000-12-07 17:07:44.5 & 2448 & $2.7_{-0.3}^{+0.4}$ & $9.0_{-1.0}^{+1.3}$ \\
\hline 33 & $50085-01-20-00$ & 2000-12-08 15:22:41.7 & 2800 & $2.5 \pm 0.3$ & $8.3 \pm 1.0$ \\
\hline 34 & $50085-01-21-00$ & 2000-12-09 16:53:56.8 & 2528 & $2.7_{-0.3}^{+0.5}$ & $9.0_{-1.0}^{+1.7}$ \\
\hline 35 & $50085-01-22-00$ & 2000-12-10 15:07:56.1 & 2880 & $2.5 \pm 0.3$ & $8.3 \pm 1.0$ \\
\hline 36 & $50085-01-23-00$ & 2000-12-11 16:39:04.3 & 2608 & $3.2 \pm 0.3$ & $10.7 \pm 1.0$ \\
\hline 37 & $50085-01-24-00$ & 2000-12-12 14:54:09.2 & 2944 & $2.7 \pm 0.3$ & $9.0 \pm 1.0$ \\
\hline 38 & $50085-01-25-00$ & 2000-12-13 10:01:16.3 & 2704 & $2.7_{-0.4}^{+0.3}$ & $9.0_{-1.3}^{+1.0}$ \\
\hline 39 & $50085-01-26-00$ & 2000-12-14 14:44:05.3 & 3040 & $3.1 \pm 0.3$ & $10.3 \pm 1.0$ \\
\hline 40 & $50085-01-27-00$ & 2000-12-15 16:10:35.3 & 2784 & $3.3_{-0.3}^{+0.4}$ & $11.0_{-1.0}^{+1.3}$ \\
\hline 41 & $50085-01-28-00$ & 2000-12-16 17:41:46.2 & 2528 & $3.2_{-0.3}^{+0.3}$ & $10.7_{-1.3}^{+1.3}$ \\
\hline 42 & 50085-01-29-00 & 2000-12-17 20:46:32.2 & 1768 & $3.4 \pm 0.4$ & $11.3 \pm 1.3$ \\
\hline 43 & $50085-01-30-00$ & 2000-12-18 17:27:43.8 & 2608 & $3.2 \pm 0.4$ & $10.7 \pm 1.3$ \\
\hline 44 & $50085-01-31-00$ & 2000-12-19 18:53:40.1 & 2336 & $3.1 \pm 0.3$ & $10.3 \pm 1.0$ \\
\hline 45 & $50085-01-32-00$ & 2000-12-20 17:07:39.4 & 2672 & $3.1 \pm 0.3$ & $10.3 \pm 1.0$ \\
\hline 46 & $50085-01-33-00$ & 2000-12-21 18:40:44.3 & 2416 & $3.0_{-0.3}^{+0.4}$ & $10.0_{-1.0}^{+1.3}$ \\
\hline 47 & $50085-01-34-00$ & $2000-12-22$ 16:55:42.2 & 2736 & $3.4_{-0.4}^{+0.3}$ & $11.3_{-1.3}^{+1.0}$ \\
\hline 48 & $50085-01-35-00$ & 2000-12-23 20:02:39.6 & 2664 & $2.6 \pm 0.3$ & $8.7 \pm 1.0$ \\
\hline 49 & $50085-01-36-00$ & 2000-12-24 18:20:17.4 & 3248 & $3.1 \pm 0.3$ & $10.3 \pm 1.0$ \\
\hline 50 & $50085-01-37-00$ & 2000-12-27 16:19:45.7 & 2896 & $3.2 \pm 0.3$ & $10.7 \pm 1.0$ \\
\hline 51 & $50085-01-38-00$ & 2000-12-30 12:47:45.9 & 1528 & $3.9_{-0.4}^{+0.5}$ & $13.0_{-1.3}^{+1.7}$ \\
\hline 52 & $50085-01-38-01$ & 2000-12-30 14:31:56.9 & 1608 & $3.4 \pm 0.4$ & $11.3 \pm 1.3$ \\
\hline 53 & $50085-01-39-00$ & 2001-01-01 12:34:12.9 & 3296 & $3.8 \pm 0.3$ & $12.7 \pm 1.0$ \\
\hline 54 & $50085-01-40-00$ & 2001-01-02 16:21:06.0 & 4512 & $3.3 \pm 0.3$ & $11.0 \pm 1.0$ \\
\hline 55 & 50085-01-41-00 & 2001-01-03 16:17:06.7 & 2728 & $3.0_{-0.3}^{+0.4}$ & $10.0_{-1.0}^{+1.3}$ \\
\hline 56 & $50085-01-42-00$ & 2001-01-04 15:32:15.4 & 2736 & $3.3_{-0.3}^{+0.4}$ & $11.0_{-1.0}^{+1.3}$ \\
\hline 57 & $50085-01-40-01$ & 2001-01-05 07:24:44.6 & 1872 & $3.1 \pm 0.4$ & $10.3 \pm 1.3$ \\
\hline 58 & $50085-01-43-00$ & 2001-01-05 10:48:27.6 & 2112 & $2.9 \pm 0.4$ & $9.7 \pm 1.3$ \\
\hline 59 & $50085-01-44-00$ & 2001-01-06 10:24:52.4 & 2912 & $2.1_{-0.3}^{+0.2}$ & $7.0_{-1.0}^{+0.7}$ \\
\hline 60 & $50085-01-45-00$ & 2001-01-07 08:38:26.6 & 2448 & $1.5_{-0.2}^{+0.3}$ & $5.0_{-0.1}^{+1.0}$ \\
\hline 61 & $50085-01-46-00$ & 2001-01-08 08:31:10.3 & 2416 & $1.3_{-0.2}^{+0.3}$ & $4.3_{-0.7}^{+1.0}$ \\
\hline 62 & $50085-01-47-00$ & 2001-01-09 14:56:14.5 & 2848 & $1.7_{-0.2}^{+0.3}$ & $5.7_{-0.7}^{+1.0}$ \\
\hline 63 & $50085-01-48-00$ & 2001-01-10 09:57:12.7 & 2816 & $1.3 \pm 0.2$ & $4.3 \pm 0.7$ \\
\hline 64 & 50085-01-49-00 & 2001-01-11 08:15:47.5 & 2368 & $1.5_{-0.2}^{+0.3}$ & $5.0_{-0.7}^{+1.0}$ \\
\hline 65 & $50085-01-50-00$ & 2001-01-14 07:49:49.7 & 2336 & $2.3_{-0.3}^{+0.2}$ & $7.7_{-1.0}^{+0.1}$ \\
\hline 66 & $50085-01-51-00$ & 2001-01-17 13:57:52.7 & 2000 & $2.8_{-0.5}^{+0.3}$ & $9.3_{-1.7}^{+1.0}$ \\
\hline 67 & $50085-01-52-00$ & 2001-01-22 13:24:36.9 & 2000 & $2.1_{-0.3}^{+0.5}$ & $7.0_{-1.0}^{+1.3}$ \\
\hline
\end{tabular}

